\title{
Autonomía catalana y democracia española: una oportunidad histórica
}

\author{
Por Josep M. VALLES CASADEVALL *
}

\begin{abstract}
An lineas generales, los partidos catalanes se enfrentar al tópico de la aspiración partécularista a una situación privilegiada en el conjunto del Estado y aceptan una regulación general de la estructura del mismo que, gatantizando la satisfacción de las reivindicaciones catalanas al reconocimiento de su personalidad y al autogobierno, sea iguamente asequible a cualquier otra comunidad aue la reclame.
\end{abstract}

Ultimado a nivel electoral el proceso de designación democrática de los titulares de poder, sólo dos personajes ocupan altas magistraturas políticas sin haberse sometido al refrendo del sufragio: el Rey y el Presidente de la Generalitat, Josep Tarradellas. Cierto es que concurren en cada caso circunstancias harto diversas, pero ambas situaciones se han visto determinadas por la peculiar naturaleza del franquismo como edificio político y por la particular transición de aquel régimen autoritario y personal a una monarquía democrática y parlamentaria. La transición en Cataluña contiene igualmente rasgos característicos que derivan de su posición anterior al franquismo y de la evolución política que experimenta durante el mismo y, más precisamente, en los años finales del período.

Con estas notas-escritas desde Cataluña a requerimiento de la dirección de REVISTA DE FOMENTO SOCIAL-, intentamos caracterizar el punto de partida y la situación presente, en el convencimiento de que la tltimación del proceso decidirá otra vez sobre el futuro de la democracia española, cuyo destino histótico va unido a la solución satisfactoria del debate sobre la concepción de España y la estructuración de su Estado.

\section{La derrota de 1939 y la difícil reconstrucción}

Los intentos de confundir una nación española uniforme con un Ëstado unitario y centralista se hallan en el origen del contencioso histótico

(*) Profesor Doctor en Derecho Político del Departamento de Ciencia Política de la facultad de Derecho de la Universidad Autónoma de Barcelona. 


\section{J. M. VALLES CASADEVALL}

que ha impedido la convivencia cómoda de los catalanes en el sistema político español. Los obstáculos adoptan nombres y apellidos diversos al hilo de los regímenes y de las situaciones. Sólo las experiencias republicanas de 1873 y 1931 auguran la solución de la «cuestión catalara», con el reconocimiento de su personalidad colectiva traducido a las estructuras políticas. Pero 1873 es sólo una fecha y la Segunda República sucumbe al embate de quienes ponen a la autonomía catalana como uno de los motivos de la insurrección antirrepublicana.

En esta oposición catalana a la forma unitaria del «Estado-nación», convergen diversas tradiciones de pensamiento: desde el conservadurismo católico de inspiración historicista hasta el republicanismo radical de orientación federalista. Con perspectivas doctrinales diversas, la identidad colectiva-negada a nivel político-se expresa en una trama de instituciones sociales extremadamente numerosas en todos los órdenes de la vida del país: religiosas, profesionales, culturales, artísticas, deportivas, económicas, etc. Son ellas las que muy a menudo sustituyen las ausentes estructuras políticas propias y las que alimentan y complementan la acción de los movimientos estrictamente políticos. Sin tener presente este dato social, se hace difícil explicar la supervivencia-en condiciones adversasdel «catalanismo» en todas sus expresiones.

El desenlace de la guerra civil constituye precisamente uno de los más graves momentos de su historia colectiva. Porque la derrota de 1939 -comparable en la distancia a la de 1714 -tiene en Cataluña un doble contenido: junto con la pérdida de las libertades democráticas que afecta a toda España, Cataluña experimenta la negación oficial de su personalidad nacional. Esta coincidencia explicará, en buena parte, los elementos distintivos de la resistencia antifranquista catalana, puesto que el combate por la recuperación de las libertades democráticas se confundirá, reforzándola, con la lucha por la restauración de las libertades nacionales.

No por menos conocido puede dejarse a un lado el capítulo de agravios adicionales que en Cataluña significó la derrota de la República. Derogación por derecho de conquista del Estatuto de autonomía y supresión de las instituciones que la encarnaban, fusilamiento sumario de su máxima representación política, el Presidente de la Generalitat Lluis Companys, prohibición del uso oficial y público de la lengua, clausura o suspensión de buena parte de las instituciones, organizaciones y publicaciones no políticas del país, prohibición y consiguiente penalización de los símbolos colectivos (bandera, escudo, himno nacional, etc.), etc. Añádase a ello la liquidación física o civil no sólo de líderes y militantes significados por su orientación política, sino también de ciudadanos caracterizados exclusivamente por su sentimiento patriótico.

La postguerra obligará, pues, a una etapa de mera supervivencia, donde la tentación del abandono-tan vigorosamente expresada por el poeta Espriu-puso a prueba a muchos. Más adelante, será posible ya -dentro de estrechos límites-una parcial reconstrucción de instituciones colectivas en el empeño que un conocido lider describió entonces como 
«hacer pás», estableciendo cimientos sociales para mejor asentat el futuro edificio político. Es en este período cuando surgen iniciativas-fundaciones, asociaciones, simples grupos sin nombre $y$, más adelante, colegios profesionales, asociaciones de vecinos, centros comarcales, etc.-que asumen tareas claramente correspondientes a un sector público que el país no poseía. Es el momento de las actividades «de peaje», que exigen a los usuarios una contribución personal y económica adicional a un ptesupuesto estatal que ignora tales necesidades colectivas.

\section{Lucba unitaria por las libertades nacionales $y$ las libertades democráticas}

Sobre este terreno se articularán de manera progresiva los movimientos propiamente políticos, cuya actuación más o menos intensa estuvo reducida a la catacumba de toda la resistencia española al francuismo.

Lo que posiblemente caracteriza esta emergencia de las fuerzas antifranquistas catalanas es su posición anticipadamente unitaria con respecto a otros territorios del Estado. Desde 1966, en efecto, se institucionaliza de manera progresiva la coordinación entre los grupos políticos catalanes, con inclusión del PSUC-partido comunista creado en 1936 por la fusión de grupos comunistas y socialistas de ámbito nacional catalán-, arrancado del ostracismo político al que le habían arrojado las circunstancias de la postguerra. En esta labor conjunta de animación de la resistencia participan pronto por igual democristianos, socialistas, republicanos y comunistas.

Esta dinámica política catalana engendra en 1971 un órgano peculiar - - la «Assemblea de Catalunya»-en la que se dan cita, junto a los partidos y grupos políticos, representaciones de la trama institucional de que antes hablamos: desde los sindicatos a los colegios profesionales, pasando por asociaciones religiosas, culturales vecinales, comarcales, representaciones universitarias, movimientos de juventud, etc. La «Assemblea de Catalunya» en su sesión constituyente adopta un programa de cuatro puntos: amnistía, libertades democráticas, restablecimiento de la autonomía y cootdinación con la lucha democrática de los demás pueblos peninsulares.

Este programa-marco inspirará las principales acciones unitarias que se desarrollan en los años finales del franquismo, cuando al progresivo agotamiento físico de su dirigente se une el agotamiento político de la fórmula autoritaria. La lucha política pasa ya a un primer plano, donde la identificación de la reivindicación democrática con la reividicación nacional engendra una dinámica esencialmente unitaria.

En función de esta dinámica hay que entender el movimiento político catalán que sigue a la muerte del general Franco. Por un lado, existe el rechazo unánime a las propuestas de «descentralización administrativa» que el Gobierno Arias-Fraga considera suficientes para saldar una de las herencias más pesadas del franquismo. Pot otro lado, la posición cohe- 


\section{J. M. VALLES CASADEVALL}

rente de las fuerzas políticas catalanas favorece la adopción-mon parte de la oposición democrática y de sus organismos de coordinación- de una clara defensa de los derechos de las nacionalidades y regiones. Fuerzas políticas españolas históticamente poco sensibles a la radicalidad del prom blema, aceptan ahora el planteamiento que de la cuestión se hace en Ca taluña. Así se expresa en el documento de la llamada «Comistón de los 10», que, con fecha de 27 de noviembre de 1976, incluye los puntos centrales de las reivindicaciones democráticas expuestas al segundo Go bierno de la Monarquía y primero presidido por el señor Suárez.

Con este hecho, se introduce en la politica estatal un tratamiento del tema que se expresa en un triple enfoque: aceptación del término «nacionalidades» junto al de «regiones» y reivindicación de las autonomías y de las instituciones que las encarnan. Tal enfoque queda formalmente asumido por los grupos políticos democráticos del Estado en respuesta a la actitud de Cataluña, Euskadi y Galicia. Los dos áltimos extremos - recuperación de la autonomía y restablecimiento de la instituciónsignifican el reconocimiento de lo que para los partidos catalanes cons. tituye factor central de su revindicación: la legitimidad histórica de un derecho-la autonomía-y de una institución-la Generalitat-que interumpieron su vigencia y su actividad por el mero efecto de la fuerza militar.

Esta pretensión de legitimidad tiene un símbolo en la figura del Pre. sidente de la Generalitat en el exilio, Josep Tarradellas, que conserva celosamente del depósito de una continuidad cuasi-dinástica con la Generalitat republicana. El movimiento popular por el retorno del President exiliado-una figura prácticamente desconocida para las nuevas generaciones catalanas - se amplifica sorprendentemente debido a lo que contiene de signo de continuidad histórica y pot encima de connotaciones petsonales y políticas.

\section{El claro veredicto de las umas: las elecciones de 1977}

En estas condiciones se convocan las elecciones del 15 de junio de 1977. La campaña adquiere en Cataluña un carácter peculiar: no se trata únicamente de la elección entre las posiciones más o menos continuistas y actitudes decididamente democratizadoras. Se trata, muy especialmente, de detectar la conciencia del electotado ante las opciones que - sobre la futura personalidad política de Cataluña-son ofrecidas por las fuerzas en presencia.

Tales opciones se reducen a tres grandes corrientes. De un lado, la posición regionalista y descentralizadota de Alianza Popular que-ditigida por el ex ministro López Rodó no va más allá de los proyectos preparados durante el Gobierno Arias-Fraga. De otra parte, la terminante posición nacional y autonomista sustentada por los nacionalistas del Pacte Democratic de Catalunya dirigido por Pujol-, los socialistas en coalición PSCPSOE-, los comunistas del PSUC, los republicanos de Es 
querra, los democristianos de UCDCC y otros grupos menores. En posición ambigua y poco definida, se sitúa la candidatura preconizada por UCD en Catalunya que evita definiciones temminantes sobre un tema dave en la campaña electoral del país. Los grupos de vocación independentista no concurren a las elecciones.

Los resultados del 15 de junio de 1977 en Cataluna presentan, con respecto al panorata politico español, un cuadro claramente diferenciado. Tres aspectos sobresalen en dicho cuadro. En primer lagar, la distrbución del voto entre los partidos ofrece un mayor equilibrio: cuatro formaciones-socialistas, comunistas, «Pacte Denocrátic» y ucedistas-superan el 15 por 100 de los sufragios, frente a la hegemonía clara de UCD y PSOE a nivel de Estado. En segundo lugar, va decidida orientación a la izquierda: dejando a un lado otros grupos politicos que se siman también en ex extremo izquierda del mapa político, «Socialistes de Catalunya» y PSUC reúnen entre ambos el 46,6 por 100 de los sufragios. El partido del Gobietno-mayoritario a nivel de Estado-ocupa ca Cataluna el cuatto lugar del «ranking» electoral, mientras que la presencia de Alianza Popular es significativamente inferior a la conseguida en el resto de España $(3,5$ por 100 de los votos).

Finalmente - y este es el punto que aquí más directamente nos atañetales resultados constituyen un claro plebiscito en tavor de las tesis que afirman la personalidad nacional de Cataluña y se pronuncian terminantemente por la recuperación de la autonomía politica. La situación minoritaria de $\mathrm{AP}$-con su actitud hostil-y de UCD - con su posición reservada-es reveladora. Los rasgos de este cuadro se refuetzan si se tienen en cuenta los resultados de las elecciones senatoriales, en las que los candidatos que asumen las posiciones que los partidos mayoritarios se hacen con todos los escaños electivos, excepto uno.

Mas precisamente, es necesario subrayar que-en el seno de este bloque nacional y autonomista-son abora las fuerzas de izquierda socialista * comunista las que ocupan un papel mayoritario y motor. Se completa así un ciclo histórico de afirmación nacional catalana, cuyos portavoces principales han sido sucesivamente los representantes de la derecha moderada, los líderes de la pequeña burguesía radical y, en el momento presente, las formaciones obreras de orientación marxista. Digamos de pasada que se hace difícil, ante tal evidencia, el análisis simplista que reduce el fenómeno nacionalista a la exclusiva defensa de unos intereses de clase.

Desde un punto de vista de estrategia política inmediata, el hecho cobra también particular relieve. El veredicto de las urnas ha sido suficientemente claro para que el Gobierno de Madrid-que hasta entonces habia adoptado una actitud dilatoria frente a las reivindicaciones de Caraluña-, tenga que ofrecer una respuesta. Basta consultar la prensa de los días anteriores e inmediatamente siguientes a la consulta electoral para detectar la inquietud suscitada por la posible canalización de la opinión mayoritaria de los catalanes hacia algún movitmiento de hecho. 
El regreso del Presidente Tarradellas

$y$ los actserdos tripartitos

En este momento, se produce la sorpresa. El Presidente de la Generalitat en el exilio es invisado a Madrid, con un tácito reconocimiento de su legitimidad histónica. La negociación entre Suárez y Tarradellas establece un nuevo triángulo politico: Gobierno central-Presidente de la Generalitat-fuerzas parlamentarias catalanas, amortiguando la confrontación directa entre el Gobiemo central dirigido por el centronderechismo de UCD y el frente nacionalista catalán hegemonizado por la izquierda.

El regreso de Tarradellas no es un mero acto simbólico. E1 Presidente ejerce una iniciativa política propia que debe conjugarse, no sin dificultades, con la iniciativa de los parlamentarios elegidos el 15 de junio y constituidos en Asamblea. Las negociaciones tripartitas, pues, van a prolongarse tres meses, hasta culminar en la reunión conjunta de 27 y 28 de septiembre celebrada en Sant Cebriá (Catalunya francesa) con intervención de las tres partes interesadas.

Como resultado del acuerdo, un Real Decreto-Ley de 29 de septiembre restablece con carácter provisional la Generalitat de Catalunya, de la que son órganos el Presidente--nombrado por el Rey a propuesta del Presidente del Gobiemo-y el Consell Executiu o Gobienno-integrado por un máximo de doce miembros, designados por el Presidentes de la Generalitat-, a los que se añadirán. los Presidentes de las cuatro Diputaciones provinciales catalanas.

Este cuadro legal será cumplimentado con arreglo a los acuerdos del protocolo suscrito por Tarradellas y los partidos políticos, referente a la composición del Consell y a la función de la Asamblea de parlamentarios que el Real Decreto no contempla.

Con arreglo a tal protocolo, la composición del Consell se ajustará a la proporcionalidad resultante de las elecciones del 15 de junio y quedará integrado por cinco Consellers políticos sin cartera-los dirigentes de las fuerzas políticas más importantes-y siete Consellers técnicos responsables de los Departamentos y designados también con arreglo a criterios de proporcionalidad. La Asamblea de Parlamentarios no figura, en cambio, entre los órganos de la Generalitat, pero sus miembros-responsables, por otra parte, de la elaboración del proyecto de Estatuto-serán informados y consultados acerca de la actuación de la Generalitat.

De acuerdo con este esquema legal, el Presidente de la Generalitat. republicana en el exilio es nombrado Presidente de la Generalitat monárquica por Real Decreto de 17 de octubre. La gestación del Gobierno catalán es laboriosa: no se produce hasta el 5 de diciembre del mismo año. Se respeta la proporcionalidad política antes apuntada, pero se deja sin efecto de incorporación de los Presidentes de las Diputaciones debido a la oposición de los partidos que discuten su representatividad democrática. 
Simbolo y realidad en la Generalitat restaurada

Con este sucinto edificio juridico, Cataluna recupera su personalidad politica expresada de nuevo en la Generalizat restablecida. El valor simbólico del hecho no es pequeño, tras la negativa rotunda y violenta del régimen anterior. Porcule-según la opinión de los expertos y según la interpretación política que sustenta el mismo Presidente Tarradelas-no nos ballamos ante una situación totalmente nueva, sino ante la reposición. de una situación anterior interrumpida por un dilatado paréntesis histórico. En este sentido, la Generalitat-afirmarán los comentaristas del Real Decreto-Ley - ro es equiparable a los demás organismos pre-autonómicos, ya que se trata de una institución que no se crea sino que se restablece. $X$, al mismo tiempo, la designación para ocupat la Presidencia de la misma recae en quien alega el depósito de la contimuidad histórica, no derivada exclusivamente de la voluntad del podes estatal ni de la elección de los parlamentarios.

Pero tampoco se ha producido-ni podía producirse, dada la situación objetiva en que se da a nivel de Estado el paso de la dictadura a la monarquía democtática-un acto unilateral de reposición de la Generalitat en virtud de la voluntad del pueblo catalán, aun cuando esta voluntad haya quedado claramente reflejada en los resultados de junio de 1977 y en la manifestación del 11 de septiembre de 1977 , cuando al decir de los observadores se produce libremente la mayor concentración popular conocida en Europa desde la liberación de París en 1944.

Sin embargo, la vigencia de lo simbólico no arrastra consigo efectivas esferas de decisión política ni oculta las dificultades políticas del acuerdo tripartito de que antes hablamos. Por un lado, las transferencias de servicios del Estado a la Generalitat son trabajosamente negociadas y muy lentamente realizadas. Por otro, la especial relación entre el Presidente y el Gobierno de la Generalitat-gobienno de unidad, con mayoría izquierdism ta-somete la acción política a un ritmo nada intenso, controlado por el especial sistema de equilibrio entre la legitimidad personal de Tarradellas y la legitimidad electoral de los partidos. La dinámica de la sociedad catalana-en sus diversas vertientes de actividad colectiva-encuentra en la Generalitat provisional un patrocinio distinguido y una acogida cálida, pero echa de menos el impulso creador y operativo para muchas iniciativas de interés general que siguen supeditadas a la benevolencia privada.

\section{La Constitución de 1978: nación y nacionalidades}

Este compás de espera no puede desligarse de la etapa política que se inicia con la apertura de las Cortes elegidas el 15 de junio. Tras la menguada victoria del partido gubernamental, las Cortes se hacen efectivamente constituyentes y en su ámbito tiene lugar el compromiso que genera el código político fundamental de la nueva denocracia. 


\section{J. M. VALLES CASADEVALL}

Entre sus primeras cuestiones-si no la ptimera, como dejará ver la agudeza de los debates al respecto-figura la revisión del concepto de España y de su estrucuración política. El fracaso del franquismo es, en este terreno, de una deslumbtante eyidencia: no sólo subsisten en todo sta vigor-y en algún caso agravadas por la violencia armada- las revivindicaciones colectivas de algunas comunidades histónicas que reclaman el weconocimiento de su personalidad, sino que emergen con más o metros fuerza ruevas voces que disctepan de la visión unifornista y centraliza dora del Estado que el franquismo quiso imponer a toda costa.

El tema se desarrolla -a lo largo del debate constitucional-en dos registros diferentes: la expresión de la realidad pural de España y la estructuración política de la nisina. El priner nivel se expresa en la discusión sobre el término unacionalidades» y su relación con la noción de «Epaña». El segundo nivel se refleya en su repercusión político-institucional que marca la estructura estatal. No resumiremos aquí la discusión constitucional, pero sí conviene retener las principales posiciones para entender hasta qué punto afecta todo ello a la politica catalana.

Por una parte, se contraponen dos concepciones de «España»: la que la entiende como una acabada unidad nacional con peculiaridades particulares, y la que la afimma como la expresión política histórica de una realidad plurinacional. Para los defensores de la primera, no existe más na ción na nacionalidad que la española $y$, por consiguiente, se oponeh al reconocimiento nacional o «nacionalitario» de cualquier orra comunidad. Para quienes sustentan la segunda concepción, la viabilidad histórica de la unidad española sólo puede cundarse en el terminante reconocimiento de su composición plural y, pot tanto, en la aceptación de que existe más de una comunidad con personalidad nacional.

E1 debate no es nuevo, puesto que retoma los términos de la discusión sobte «la esencia» de España desde que, en el siglo XIX, la categoŕa de «nación» es reelaborada desde la perspectiva liberal. Pero tal reelaboración, hecha a partir de formaciones sociales concretas e históricas, conduce casi siempre a un tratamiento idealista de la cuestión que inpide resolver los problemas específicos de otras sociedades y, entre ellas, de la española. El resultado de un debate transportado a España en estos términos conduce a la irresoluble antinomia «imperialismo espanolista»-«separatismo catalanista, vasquista, canario, etc.»-, que no tiene más solución política que la impuesta por la «violencia estatal» o por la «violencia terrotista».

La utilización del térnino nacionalidad-adoptado previamente en la literatura política y en los programas de los partidos antifranquistas constituye un intento de salir del impasse nominalista anterior, con la in. tención de aproximar las postutas de quienes ven a una España «nacional» con diversidad de tegiones y de quienes, en cambio, considetan a España como la resultante de una diversidad de naciones. La expresión «España, nación de naciones -avanzada por un polítco de UCD-sería igualuente una tertativa para romper la rutina de los clisés heredados. 


\section{AUTONOMIA CATALANA}

La discusión constitucional se resolvio - como es sabido-en la redacción del art. 2, donde se incluye, por vez primera en los textos fundamentales españoles, el término nacionalidades. Tal inclusión viene compensada por afirmaciones rotundas y reiterativas sobre la unidad de la nación española y por la invocación-insólita en la literatura constitucional-a «la patria común e indisoluble de todos los españoles». La redacción abarrocada del citado artículo responde a la sucesión de intervencio. nes que experimentó y que no se linitaron al ámbito patlamentario, puesto que-según se afirmó en la prensa-influyeton de algín modo en la redacción algunas indicaciones de sectores militares.

Con todo, la constitucionalización de las nacionalidades-aungue de modo un tanto vergonzante, puesto que el término no aparece más que una sola vez en todo el texto-es un paso adelante en la construcción de una plataforma de entendimiento entre las diversas concepciones de España que subsisten en comunidades de su ámbito de referencia histónico.

Más en concreto, para la opinión catalana-que, en su gran mayoría, no se reconoce en el tratamiento floklorista de las epeculiaridades regionales» de la literatura franquista-, el artículo $20^{\circ}$ es un modesto, pero positivo paso adelante en la resolución del contencioso histórico que tanto ha costado a España en su conjunto como a la misma Cataluña.

\section{La Constitución de 1978: un Estado de autonomias}

El segundo aspecto-la traducción en estructuras políticas de aduella concepción nacional-confluye, por un lado, en el debate sobre la soberanía y, por otro, en la concreta regulación del estatuto y organización de las comunidades. La posición mayoritaria de los partidos catalanes--socialistas, comunistas y «convergentes»-se sitúa entre una vocación federalista y una apreciación realista del ámbito de autogobietno asequible en las presentes circunstancias políticas generales.

Durante la dictadura y, más especialmente, en los momentos finales del franquismo, la aspiración unitaria de la oposición democrática tiene como horizonte mínimo el delimitado por la Constitución republicana de 1931 y el Estatuto de 1932 aceptados en su día a pesar de las limitaciones que impusieron a la voluntad inicial de los catalanes.

Los debates constitucionales de 1977-1978 conformarán finalmente un Estado unitario - en el que la soberanía corresponde al pueblo español (art. 1,2)-que reconoce y garantiza constitucionalmente el derecho a la autonomia de las nacionalidades y regiones que integran España (artículo 2). El alcance de la autonomía queda perfilado en el Título VIII de la Constitución.

La activa participación de los catalanes en las discusiones constitucionales sobre el tema especialmente la de los diputados Martín, Solé Turá y Roca Junyent, por los socialistas, comunistas y convergentes, respectiva- 
mente-pone de relieve que la opinión catalana se halla convencida-tal vez más que en otro tiempo-de que la resolución de la ucuestión catalana» pasa por una intervención activa en el planteamiento de la organización general del Estado. En lineas generales, los partidos catalanes se enfrentan al tópico de la aspiración particularista a una situación privilegiada en el conjunto del Estado y aceptan una regulación general de la estructura del mismo que, garantizando la satisfacción de las reivindica. ciones catalanas al reconociniento de su personaldad y al autogobieno, sea igualmente asequible a cualquier otra comunidad que la reclame.

No es el momento de entrar en el análisis detallado del complejo Título VIII, criticado a veces por algunos «técnicos» en función de categorías jurídicas posiblemente más ortodoxas, pero probablemente mucho menos eficaces para enfrentarse con una cuestión que desborda el campo de lo meramente administrativo y contiene una carga política que es necesario subrayar aquí.

Lo que importa destacar es por qué motivos los partidos catalanes «nacionalistas»-que son, en virtud de su programa, socialistas, comunistas, convergentes y democratacristianos han aceptado la solución constiw tucional desde la perspectiva catalana. Al entender de tales partidos, la Constitución hace posible-tras la aceptación de las nacionalidades y de su derecho a la autonomia-la recuperación de una esfera de autogobierno que se delimita con intervención concurrente de dos voluntades: la emanada de la representación estatal y la derivada de la representación de la comunidad afectada. Dos son, pues, los aspectos clave de la cuestión: el primero afecta directamente a la amplitud de las competencias posibles y a la obtención de los recursos financieros para gestionarlas; el segundo, corresponde al procedimiento de aprobación y reforma del Estatuto con arteglo al cual se establece la situación de la comunidad autónoma.

In ambos aspectos, la solución final aparece como razonablemente satisfactoria para las aspiraciones catalanas $y$, al mismo tiempo, no entra en contradicción con otzas posibles dinámicas en el interior del Estado. De otra manera: la Constitución no prevé las temidas «autonomáas de primera y de segunda», salvo en el ritmo de adquisición de competencias y recursos que se moverán en todo caso en los mismos límites.

La disposición transitoria segunda es únicamente una nueva expresión de la particular transición de un régimen anterior-que negaba todo pasado histórico que desmintiera su visión del mundo y de la realidad española-y la nueva situación democrática-que se reconoce en alguna medida en una tradición democrática y liberal no por agitada menos valiosa. En este punto, la preexistencia de Estatutos de autonomía en virtud de la legalidad constitucional tepublicana es reconocida como punto de arratuque aventajado para iniciat un proceso que, sin embargo, no tiene otro punto de llegada que al ofrecido a todas las comunidades del Estado. 


\section{El proyecto del Estatuto de 1978 y las elecciones de 1979}

Con la aprobación de la Constitución queda abierto el nuevo periodo estatuyente. Los parlamentatios catalanes se aplican a la elaboración del texto básico de su autonomía que será sometido sucesivamente a la hegoctación con la Comisión constitucional de las Cortes, a la consulta re ferendaria del pueblo catalán y, finalmente, a la eventual tatificación de parlamento español. El proyecto de Estatuto es aprobado oficialmente el 29 de diciembre de 1978, por los diputados y senadores de Cataluña con una sola abstención y ningún voto en contra. Durante los debates, sólo una cuestión provoca un claro enfrentamiento: la referida al sistema electoral provisional previsto para el Parlamento de Cataluña. Socialistas y comunistas, por un lado, y convergentes y ucedistas, por otro, discrepan en cuanto a la circunscripción electoral. Para los primetos, provisionalmente- $y$ en tanto no se proceda a una nueva división territorial en Cataluña-debe adoptarse la circunscripción electoral única o, en su defecto, las actuales circunscripciones provinciales; para los segundos, y también de modo provisional, debe optarse por la comarca-de honda raíz social-como distrito electoral. Como en toda discusión normativa sobre elecciones, cada fuerza política sostiene como más interesante la posición técnica que le beneficia en el proceso de convertir los votos populares en representantes parlamentarios. Es, pues, una lógica discrepancia debida a la diferente implantación territorial de cada una de las grandes formaciones políticas catalanas: socialistas, comunistas, centristas-UCD y convergentes El desenlace de la discusión apunta a un relativo compromiso, al establecer como distrito electoral provisional una unidad intermedia entre la circunscripción única y la circunscripción comarcal: se trata de la región resultante de la agrupación de comarcas, con arreglo a la división territorial establecida por la Generalitat republicana en 1936.

Salvo este punto, que afecta a la posición interna en Cataluña de los intereses de cada partido, el texto se mueve en los límites marcados pos la Constitución, aspirando a la mayor amplitud de competencias y a la mejor situación en cuanto a los recursos. Por lo que a las instituciones se refiere, se reproduce en líneas generales el esquema de la Generalitat de 1932, con un régimen parlamentario que hace al Presidente y a su Gobieno responsable ante la asamblea. Queda a la práctica política del decidir hasta qué punto el centro de decisiones politicas en el seno del Ejecutivo corresponderá al Presidente o a un posible «primer ministro» delegado, dilucidando así otro de los puntos que ocasionó un cierto contraste de posiciones.

El proyecto de Estatuto llega a las Cortes elegidas en 1977, cuando el Presidente Suárez ha anunciado ya su disolución y la convocatoria de nuevos comicios para el mes de matzo de 1979. El calendario político se interfiere, pues, con el proceso de elaboración del Estatuto y los resul. tados de las elecciones se convierten naturalmente, en un factor altamente condicionante de dicho proceso.

Las elecciones del $1 .^{\circ}$ de marzo no producen en Cataluña sustanciales 


\section{J. M. VALLES CASADEVALL}

modificaciones del panorama politico que presenta nuevamente el modelo cuatripartito perfilado en junio de 1977. Se mantiene-y aumenta ligeramente-el primer lugat del PSUC, aumenta ligeramente UCD que pasa a ocupar el segundo hugar del «ranking» electoral catalán peto todavía a gran distancia de los socialistas, y, finalmente, ligero descenso del PSUC y de CDC que se sitúan, respectivanente, en tercer y cuarto lugar.

Sin embargo, unas semanas después, las elecciones municipales del 3 de abril señalarán para Cataluna un notable avance de la izquierda en su conjuntom-y, especialmente, de los comunistas-, un fortalecimiento de CDC y un considerable retroceso de la coalición «Centristas-UCD», que refleja su escaso arraigo en los núdeos locales. El poder local se estructura a partir del pacto tripartito PSC-PSUC-CDC, que ásla a UCD y la sitúa-salvo en algún ayuntamiento de menos de mil habitantes-men la oposición municipal. La recuperación electoral de los partidos nacionales que, por vez primera en esta transición, acceden ahora a posiciones de poder-si bien limitado a municipios y diputaciones-introduce un nuevo elenento en la dinámica hacia la autonomía.

Cierto es que la tramitación del Estatuto no pasa, desde el punto de vista juridico, por los niveles de la administración local. Pero los partidos que la controlan y que son también los más decididos impulsores de la recuperación del régimen autonómico hatán sentir desde ella un sentimienco general, expresado de nuevo en la manifestación popular por la recuperación del Estatuto, celebrada el pasado 23 de abril, a pesar de las cautelas manifestadas por el Presidente de la Generalitat y de la no adhesión de UCD, único partido que no se sumó a la convocatoria.

\section{Claroscuro del próximo debate estatutario: una nuevo oportunidad bistórica}

Con la discusión parlamentaria del proyecto de Estatuto, prevista para los meses de verano y otoño, se enttará en una tercera y definitiva etapa del proceso. Tal discusión va a celebratse cuando se ha reforzado en Cataluna la posición de las fuerzas nacionales y autonómicas, mientras que la mayoría politica en el parlamento del Estado sigue orientada a la derecha. Es cietco que tal mayoría moderada se encuentra probablemente en mejor actitud que las formaciones de la derecha histórica de otro tiempo. Con todo, existen indicios de que la mayoría gubernamental puede caer en la tentación de dar a los debates un titmo premioso y marcado por limites que podrían desvirtuar el significado político del consenso constitucional. Afortunadamente, la tentativa de considerar decaidos los proyectos de Estatuto vasco y catalán-presentados antes del término de la anterior legislatura han sido superados con la tatificación de los citados textos como base de un debate que no puede aplazarse.

En cambio, el empeño del Gobierno por anteponer a la discusión de los Estatutos la aprobación de algunas leyes orgánicas como, por ejemplo, las referidas a financiación de las autonomías o a la enseñanza y orga- 
nización universitaria, representara-de prospetar los proyectos tal como han sido conocidos-un serio recorte e incluso una destiación de las previsiones fundadas en el Título VIII de la Constitución.

Ni la lentificación del proceso ni el tratamiento cicatero del contendo del proyecto responderían a la importancia de una cuestión que tiene que ser contemplada en un horizonte más ancho que el marcado por la esca. ramuza politica del momento. La traducción de la nomativa constitucional a un régimen autonómico satisfactorio es una oportunidad histórica para resolver de forma dutadera un pleito secular, en el que a menudo no fueron los pueblos quienes se enfrentaron, sino los intereses de grupos sociales minoritarios apoyados en el instrumento del Estado unitario y centralista para la consolidación y defensa de sus intereses. Toy, las fuerzas de izquierda-socialistas y comunistas-del Estado han incorporado a sus propuestas la revisión de aquella forma politica tar poco ventajosa para los intereses populares. Por su parte, sectores amplios de la bur guesía coinciden en la necesidad de una reforma del testado y de la administración que los sitúe en niveles más racionales de eficacia, sin menoscabo de los intereses fundamentales que defiende. Todo ello define un terreno de coincidencia, que ofrece un mejor eco a la reivindicación persistente de Cataluña.

No habrá que descuidar tampoco la posición más reticente-y de influencia nada despreciable-mantenida por sectores de la misma Administración que, aduciendo motivos técnicos, defienden parcelas importantes de su condominio sobre el Estado capitalista moderno, en el que algunos colectivos de funcionatios se encuentran a caballo de la gestión pública y privada de los intereses dominantes.

Por estas y otras razones, no será extraño que el debate estatutario de los meses venideros ofrezca momentos difíciles para los negociadores. En tal caso, no debería ser engañosamente interpretada la moderación con que se han producido hasta ahora la opintón. pública catalana y sus representantes políticos. El clamor pacífico por el reconocimiento efectivo de su personalidad nacional ha sido prácticamente unánime, a través de las generaciones y con independencia del origen de sus ciudadanos. Des. atender ahora este clamor sería perder la ocasión para consumar al mismo tiempo un acto de justicia histórica y una muestra de inteligencia política, olvidando nuevamente que el destino de la democracia española ha estado unido en buena parte al concurso decidido de una Cataluña identificada consigo misma y reconciliada con los demás pueblos de España. 